\title{
The art of coming home. Experiencias de reinserción laboral de migrantes deportados de Estados Unidos
}

\author{
The art of coming home. Labor reintegration \\ experiences of deported migrants from United States
}

Recibido el 22 de abril de 2020.

Aceptado el 6 de octubre de 2020.

Publicado el 13 de octubre de 2020.

*Autora para correspondencia: Ana Elizabeth Jardón Hernández. Correo electrónico: ileana.14@hotmail.com

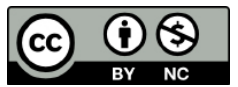

Esta obra está protegida bajo una Licencia Creative Commons Atribución-NoComercial 4.0 Internacional.

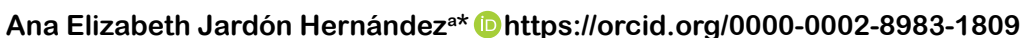
Zoraida Ronzón Hernándeza (i) https://orcid.org/0000-0003-3597-3710

\footnotetext{
a Universidad Autónoma del Estado de México, Centro de Investigación en Ciencias Sociales y Humanidades, Toluca, Estado de México, México. Correo electrónico: ileana.14@hotmail.com, zoraronzon@hotmail.com
}

\section{Resumen}

El objetivo del artículo es analizar la experiencia de la deportación y reinserción laboral de una mujer migrante que se ha establecido en el municipio de Nezahualcóyotl, Estado de México desde 2016. A partir de una metodología cualitativa y un enfoque biográfico recuperamos la voz de nuestra informante y su subjetividad sobre la experiencia vivida en Estados Unidos, así como de las acciones emprendidas para autoemplearse en un contexto urbano. Los hallazgos advierten que entre la población migrante deportada es necesario cambiar la mirada del norte al sur para una reinserción laboral exitosa, aunque ello no necesariamente signifique que pueden despojarse del que consideran su hogar en ese país. Asimismo, se destaca el papel de la persona migrante como agente y de los capitales humano, social y político como recursos para la implementación de iniciativas que promueven la reinserción laboral individual y colectiva de los migrantes deportados.

Palabras clave: deportación, reinserción laboral, Colectivo Deportados Unidos en la Lucha, retorno, migración.

\section{Abstract}

This article aims to analyze the experience of deportation and labor reintegration of a migrant woman who has settled in the municipality of Nezahualcóyotl, State of Mexico since 2016. Using a qualitative methodology and a biographical approach, we recovered the voice of our informant and her subjectivity on the experience lived in the United States, as well as the actions taken to self-employ in an urban context. The findings warn that among the deported migrant population, it is necessary to change the gaze from north to south for a successful reintegration into the labor market, without necessarily implying what they consider their

CÓMO CITAR: Jardón Hernández, A. E. \& Ronzón Hernández, Z. (2020). The art of coming home. Experiencias de reinserción laboral de migrantes deportados de Estados Unidos [The art of coming home. Labor reintegration experiences of deported migrants from United States]. Estudios Fronterizos, 21, e058. https://doi.org/10.21670/ref.2016058 
home in that country. Likewise, the role of the migrant person as agent and of human, social and political capital as resources for the implementation of initiatives that promote individual and collective labor reintegration of deported migrants is highlighted.

Keywords: deportation, labor reintegration, Deportees Together in the Battle Organization, return, migration.

\section{Introducción}

Yo sigo diciendo yo estoy aquí [en México], pero mi casa, que yo diga es mi casa, luego hasta me siento mal porque no sé qué decir ya ¿̇es mi casa, no es mi casa? es difícil decidir, pero mi casa es en Chicago, yo sí sigo viendo mi casa en Chicago, aquí estoy adaptándome, pero mi concepto, lo que yo entiendo de hogar está en Chicago (Laura, Estado de México, enero de 2020).

Analizar los procesos de deportación de la población mexicana desde una mirada histórica permite comprender, por ejemplo, que la población que regresó a México durante la gran depresión (1929-1934) lo hizo en condiciones que no resultan del todo ajenas a los retornados en las administraciones de Obama y Trump, toda vez que el retorno masivo y apresurado de las miles de personas que volvieron a México en esos años también estuvo vinculado con situaciones de desempleo, hambre, xenofobia y deportaciones (Alanís, 2005). Por ello, al inicio de este trabajo presentamos un breve fragmento de la entrevista realizada a Laura, una mujer migrante deportada de Estados Unidos — cuyo caso exponemos con amplitud en el desarrollo de este artículo-, con la finalidad de poner en contexto algunas de las implicaciones que la legislación migratoria de Estados Unidos ha tenido y tiene en la vida de las personas migrantes, particularmente ante la implementación de acciones violentas que buscan "vigilar y castigar, excluir, negar, rechazar y deportar, [...así como] proteger la frontera y criminalizar al otro" (Castañeda, 2012, pp. 301-312).

La deportación, como lo advierte Padilla (2012, p. 212), es "un ejemplo de la manera en que las leyes migratorias estadounidenses crean infractores y criminales". Regresar con esa etiqueta, muchas veces en condiciones de vulnerabilidad, no hace sencilla la reinserción en los contextos de llegada en México, donde habrán de diseñar estrategias para incorporarse a la dinámica de esos lugares. En algunos casos de forma temporal, mientras que en otros de manera permanente por las condiciones en las que ocurrió el regreso forzado, las sanciones asociadas a este y las muchas vulnerabilidades que suponen las entradas ilegales a ese país.

Nuestro trabajo tiene por objetivo exponer la experiencia de la deportación y de reinserción laboral de una mujer migrante que se ha asentado en una zona urbana del Estado de México. Esto ante un escenario en el que la criminalización de la migración ha hecho que "los migrantes pasen de ser trabajadores, padres, madres e hijos, a ser criminales, [con lo que...] adquieren una nueva subjetividad con secuelas graves para sus vidas y las de sus familias" (Castañeda, 2012, p. 309). Ejemplo de ello es la separación familiar, que en algunos migrantes deportados recrea luchas constantes entre la búsqueda por reinsertarse en México y la esperanza de regresar a Estados Unidos para estar en la que consideran su casa. 
De ahí la noción plasmada en el título de nuestro trabajo: "The art of coming home" retomada de la obra de Storti (2011, en u.s. Department of State, 2017), con la finalidad de precisar, según lo establece este autor, que la casa no es propiamente el lugar físico que habitamos, sino el espacio asociado con todas las personas, las acciones, los sentimientos, las emociones y las señales que nos hacen sentir en casa. Así lo expresa Laura, nuestra informante, se trata de todo lo que construye tu vida, pues "es tu vida la que está ahí” para referirse a su hogar en Chicago, Illinois, Estados Unidos.

La interrogante a la que buscamos dar respuesta a través del caso presentado en este trabajo es: ¿cómo interpretar los procesos de reinserción laboral de población migrante deportada en contextos urbanos de llegada en México? Para tal efecto, el artículo se integra de cuatro apartados. En el primero se expone la metodología empleada y las técnicas de recolección y análisis de la información. Posteriormente se presentan los principales referentes conceptuales para entender los procesos de reinserción laboral. En el tercer apartado, sustentado en la narrativa de Laura, se describe y analiza su experiencia de deportación y los procesos de reinserción con establecimiento en el municipio de Nezahualcóyotl, Estado de México. Finalmente presentamos las principales conclusiones de este trabajo.

\section{Metodología}

De acuerdo con Castles (2012), comprender la relación entre metodología y métodos es necesario en la medida en que generalmente son confundidos o utilizados como si ambos significaran lo mismo. Mientras que la metodología suscribe la lógica de la investigación y está vinculada con la teoría del conocimiento, los métodos son las técnicas específicas que utilizamos para recolectar y analizar la información o los datos.

En otras palabras, al retomar a Taylor y Bogdan (1984, p. 15), "la metodología designa el modo en que enfocamos los problemas y buscamos las respuestas". Al respecto, esta investigación cualitativa parte de una perspectiva fenomenológica, toda vez que buscamos comprender las acciones de las personas desde su propio punto de vista y/o de la forma en que definen su mundo, a decir en nuestro caso, las acciones promovidas por la población deportada de Estados Unidos para reinsertarse laboralmente y establecerse en México.

Dentro de los diferentes métodos cualitativos existen los considerados en la perspectiva biográfica (Pujadas, 1992), y entre estos encontramos las narrativas autobiográficas, que por sus particularidades visualizamos como un método adecuado para esta investigación, dado que recuperan elementos de la historia de vida que fueron elegidos dentro de la construcción misma de la investigación, con variables claramente definidas en las entrevistas (Santamarina \& Marinas, 1994), que nos permitieron incorporar la voz del informante, su subjetividad y sus valoraciones.

Las narrativas autobiográficas permiten conocer aspectos de la vida de las personas que son pertinentes a los objetivos de la investigación, además de mostrar los constructos socioculturales de hombres y mujeres alrededor de la experiencia de la deportación con sus propias palabras. Sin embargo, Velasco y Gianturco (2015, p. 136139) señalan que el uso de este método en los estudios sobre migración internacional requiere considerar la dificultad para captar la experiencia de vida constituida por lugares tan diversos que refieren distintos contextos de significados, que son diferentes 
del contexto situacional de producción del relato de vida y que determinará su fluidez, profundidad y amplitud.

En segundo término, la articulación del tiempo y espacio en las biografías de personas migrantes supone considerar que la experiencia de los tiempos está acompañada de una experiencia del tiempo fragmentado en términos geográficos y de relaciones sociales, que se expresan en el "aquí" y "allá" para dar cuenta de eventos simultáneos y conectados significativamente. Finalmente, las autoras señalan la dificultad de registrar las vidas en la clandestinidad, toda vez que la memoria, como recurso principal para reconstruir relatos de vida es una facultad que olvida, particularmente cuando se trata de situaciones con carga emocional negativa, por lo que pueden quedar en el olvido.

A partir de lo anterior, justificamos la selección del caso de Laura sobre la base de dos particularidades. En primer lugar, se trata de una mujer migrante que no es originaria de la entidad mexiquense, pero que buscó establecerse en el municipio de Nezahualcóyotl por su cercanía con la Ciudad de México y las oportunidades laborales que esta ofrece. Metodológicamente, como lo sostiene Rivera (2013, p. 56), el análisis de este tipo de casos permite "tomar distancia de las investigaciones que consideran como unidades de análisis y de referencia solo un lugar de origen y un lugar de destino para estudiar el proceso migratorio, y en consecuencia asumen que el retorno ocurre necesariamente al lugar de origen". En segundo lugar, por tratarse de una migrante retornada con un alto capital social y político, así como con una amplia iniciativa que le ha permitido convertir dichos capitales en acciones colectivas.

Nos interesa también mencionar que con este estudio de caso resaltamos la necesidad de aprender y comprender la problemática en cuestión a partir de la cercanía a los sujetos y los contextos; es decir, destacamos el potencial que el análisis a profundidad tiene para la comprensión de los procesos sociales desde la voz de nuestros sujetos de estudio, cuya especificidad no siempre se separa de la posibilidad de establecer semejanzas con lo observado en otros casos (Flyvbjerg, 2004).

El primer contacto con Laura se realizó en noviembre de 2017 a través de la organización transnacional Instituto de Investigación y Práctica Social y Cultural (Iipsoculta). En ese año, habiendo transcurrido poco más de doce meses de su deportación, Laura se estaba iniciando en el desarrollo de un proyecto productivo. En enero de 2020 recuperamos el contacto con el fin de profundizar en el conocimiento y comprensión de su experiencia vivida durante la deportación, pero particularmente con el propósito de conocer los alcances de su proyecto productivo como estrategia para la reinserción laboral.

A modo de consideración debemos señalar que las acciones promovidas por Laura después de su deportación han sido documentadas por diversos medios de comunicación, por lo que, aunque es difícil procurar el anonimato de nuestra informante, tenemos su consentimiento para recuperar y analizar la información que nos fue proporcionada.

\section{Premisas básicas sobre la deportación y la reinserción laboral}

De acuerdo con la Organización Internacional para las Migraciones (OIM), el retorno forzoso se refiere al "regreso obligado de una persona al país de origen, de tránsito o 
un tercer país, fundado en una decisión administrativa o judicial” (oIM, 2006, p. 61), entre las que la deportación es una de ellas, por tratarse de un acto del Estado para enviar a un extranjero fuera de su territorio.

De acuerdo con Castañeda (2012, p. 308), esta acción que resulta de la aplicación de una política pública se vuelve real cuando los migrantes la encarnan y la experimentan en su propio cuerpo, en el que la deportación se construye como un acto que expresa y activa la violencia y la coerción contenida en la legislación migratoria. Al mismo tiempo, sostiene la autora, la dinámica y magnitud que alcanzan estos eventos responden a contextos económicos específicos, como a sentires y malestares sociales e intereses políticos concretos, como los observados en las diferentes etapas en la historia migratoria México-Estados Unidos, con crisis económicas y posicionamientos antiinmigrantes que asignan particularidades específicas a los flujos de ida y vuelta entre ambos países (Durand, 2013; Massey, Pren et al., 2009; Massey, Durand et al., 2009).

En la subjetividad de las personas migrantes, la deportación puede representarse en un conjunto de diversas experiencias, entre las que Beltrán (2019, p. 138) identifica la deportación como una tragedia; un adelanto a los planes del retorno; una prueba de vida o de fe; una consecuencia derivada de errores individuales; un proceso legal justo, o bien, una experiencia de maltrato y violación a los derechos humanos. Experiencias que en nuestra opinión no son excluyentes la una de la otra, toda vez que, en la vivencia de su propio proceso, las personas deportadas pueden ubicar y reconocer haber vivido más de una.

Al evento de la deportación al lugar de origen u otro lugar le sucede la reintegración que la misma orm define como la "reinclusión o reincorporación de una persona a un grupo o a un proceso, por ejemplo, de un migrante en la sociedad de su país de origen" (oIm, 2006, p. 62). Entre sus tipos distingue la reintegración social, cultural y económica, de las cuales nos interesa destacar algunos aspectos para profundizar en el ámbito económico y los procesos laborales.

Por su definición, la reintegración social refiere al proceso de reinserción de una persona migrante en la estructura social de su país de origen, que incluye tanto los vínculos personales (familia, parientes, vecinos), como la sociedad civil (asociaciones civiles, grupos autónomos y otros) (оIM, 2006). En otras palabras, la reinserción social tiene un estrecho vínculo con la presencia de capital social entre estas personas, en la medida en que se trata de un recurso utilizable, que se acumula y reconvierte para desarrollar diversas prácticas que permitan resolver y atender las necesidades cotidianas (Gutiérrez, 2004).

Al seguir con la propuesta analítica de Gutiérrez (2004), el recurso como capital se expresa en forma individual, familiar y colectivo. El primero es puesto en marcha de manera individual e independiente por las personas, distinguiéndose, por ejemplo, al momento de buscar un trabajo. Mientras que el colectivo se expresa de manera institucionalizada y otras formas de organización, que en este caso pueden facilitar la reintegración social de las personas deportadas.

Por otra parte, la reintegración cultural, según la oIm (2006) tiene que ver con la readopción de los valores, formas de vida, idioma, principios morales, ideología y tradiciones del país de origen por el migrante que ha regresado. Al respecto, consideramos pertinente señalar que la readopción de dichos elementos no siempre es sencilla, ni mucho menos automática, por lo que puede resultar en lo que algunos autores (Storti, 2011, en U.s. Department of State, 2017) han denominado choque 
cultural inverso, para referirse a los impactos que tiene en la subjetividad de la persona retornada adaptarse a la cultura de su país después de haber estado un tiempo fuera de este. Tiempo en el que no solo cambió el lugar, su gente, sino la propia persona que está de vuelta y que habrá de reinsertarse a una vida que incluso puede resultarle más nueva que la que ha dejado atrás (Sanz, 2016); o bien, a un espacio de vida que no solo puede resultarle ajeno, sino que propiamente ya no constituye el hogar o la casa de la persona retornada (Storti, 2011, en U.s. Department of State, 2017).

En este proceso de readaptación inversa, una vez que se está de regreso en el país de origen, la persona experimenta un proceso semejante al que vivió en el país extranjero, con tiempos de éxtasis al encontrarse con una nueva cultura y/o reencontrarse con la suya, crisis derivadas del sentirse ajeno o extranjero, así como tiempos de recuperación y de ajuste y adaptación. Momentos que según Gullahorn y Gullahorn (2013) hacen que, en la conjugación de sentimientos y emociones asociados, primero con la emigración y después con el retorno, se experimenten altas y bajas, que pueden relacionarse con la sensación de encontrarse en una montaña rusa, con subidas y bajadas, que en nuestra opinión son cíclicas, pues con el regreso, particularmente a un espacio no asociado a la construcción del hogar, las personas pueden experimentar diversas crisis, como procesos de recuperación y esfuerzos constantes de adaptación.

De acuerdo con Storti (2011, en U.s. Department of State, 2017), algunos elementos que pueden hacer más o menos tenso este proceso tienen que ver con la modalidad del regreso (voluntario o involuntario), su planificación (esperado o inesperado), la edad, el tiempo de estancia en el extranjero, el grado de interacción con la cultura extranjera y el de interacción con la cultura de su país de origen. Factores que en su conjunto pueden ser explorados entre la población migrante deportada, cuyo regreso ha sido involuntario e inesperado después de haber permanecido un amplio periodo en Estados Unidos, que les permitió construir proyectos de vida y afianzar su pertenencia en ese país más que en México.

En otro orden de ideas, la reintegración económica, que es aquella en la que nos interesa priorizar en este artículo, tiene que ver con la reinserción de la persona migrante al sistema económico de su país de origen, con el fin no solo de garantizar su sustento, sino de propiciar el uso de los conocimientos, habilidades y activos que adquirió en el extranjero para favorecer el desarrollo de los lugares en los que se encuentra de vuelta (oIM, 2006). Desde este enfoque, pareciera entonces que la posición de la persona migrante como promotor del desarrollo queda circunscrita tanto a sus contribuciones económicas mientras estuvo en el extranjero como migrante activo, bajo la figura del migrante colectivo (Moctezuma, 2008), como también a su regreso al país de origen, bajo la presunción del uso y aprovechamiento de los conocimientos y habilidades adquiridas. Aunque esto no siempre es posible por las características de los mercados laborales en México, pues "no todos encuentran dónde, ni cómo ejercer sus conocimientos laborales. Siendo la queja más sentida de los migrantes: no vivir de lo que saben hacer" (Salas et al., 2019, p. 16).

A pesar del protagonismo que le ha sido asignado al migrante como promotor del desarrollo, cabe señalar que "los migrantes retornados no son una población objetivo en los diseños de política migratoria o de política social propiamente" (Rivera, 2011), pues en la práctica lo que se ha promovido son iniciativas aisladas e improvisadas que no siempre buscan la reintegración, como la estrategia "Somos Mexicanos" o el programa "Repatriados Trabajando", cuyos alcances en materia de colocación e 
inserción laboral fueron mínimos durante el tiempo en el que se implementaron (Jardón et al., 2019).

Específicamente, la reinserción laboral es definida como el "proceso posterior al retorno durante el cual los emigrantes no solo retornan y se reincorporan a la comunidad de la que salieron, sino que buscan dar continuidad a su trayectoria laboral a través de actividades dirigidas a la búsqueda de empleo o bien a la instalación de pequeños negocios en el lugar de origen" (Anguiano-Téllez et al., 2013, p. 117). Algunas reflexiones sobre el concepto nos permiten advertir que la reinserción no necesariamente ocurre en el que fuera el lugar de salida, en tanto la población retornada puede valorar la búsqueda de oportunidades en otros espacios, con el propósito de aplicar sus conocimientos y desarrollar sus capacidades. Además de que la instalación de pequeños negocios, aunque es una de las prácticas a la que acuden en mayor medida los migrantes retornados, no siempre tiene el alcance y rendimiento esperado (Jardón et al., 2019; El Colegio de Tlaxcala A. C. \& Gobierno del Estado de Tlaxcala, 2017). En tal sentido, se asume que para enfrentar las muchas dificultades asociadas con la reinserción laboral o el emprendimiento "se requiere de imaginación, inventiva y capital, tanto económico como humano” (Rivera, 2011, p. 330).

La reinserción laboral de los migrantes retornados, vista desde la migración como un proceso, en el que el retorno no es definitivo ni permanente, permite entender que reemigrar constituye una posibilidad siempre presente en caso de que la reinserción laboral —en este caso, en México- no represente la satisfacción de sus necesidades y expectativas familiares e individuales. Aunque en otros casos puede tratarse de un proceso más bien orientado al establecimiento (Rivera, 2011, p. 328; 2013, p. 72). Esta última modalidad de reinserción es la que nos interesa interpretar en este artículo a partir del caso de Laura. Para tal efecto, nuestro posicionamiento corresponde a la comprensión de la migración como un proceso cíclico y abierto, en el que la posibilidad de volver a emigrar estará siempre presente, incluso entre aquellos migrantes que fueron deportados con sanciones de no reingreso a Estados Unidos de por vida.

En este contexto emergen modelos orientados a propiciar la reincorporación social, laboral, económica, política, cultural y familiar de la población migrante de retorno en México. Desde una perspectiva integral, la propuesta de Rendón y Wertman (2017, p. 33) se sustenta en tres enfoques complementarios: $i$ ) derechos; ii) readaptación social-riesgo, necesidad y capacidad de respuesta; y, iii) gestión de redes.

El enfoque de derechos tiene como propósito constituir un marco de respeto, protección y adopción de medidas activas para poner en práctica leyes, políticas y procedimientos, entre las que se incluye la asignación de recursos para la procuración de los derechos de esta población migrante, como por ejemplo el derecho de nacionalidad, la no discriminación, el no ser criminalizado, salud, trabajo, entre otros, que garanticen la integridad y vida digna de los retornados, pues como ha sido documentado por diversas organizaciones de la sociedad civil (Instituto para las Mujeres en la Migración [Imumi], Instituto de Estudios y Divulgación sobre Migración [Inedim], Sin Fronteras, Iipsoculta) se trata de un conjunto de derechos básicos que no siempre son garantizados.

El segundo enfoque de este modelo de reintegración parte de considerar que el nivel de atención que recibe un migrante retornado debe ajustarse a su nivel de riesgo. En otras palabras, se asume que los migrantes en condiciones de mayor vulnerabilidad precisan de niveles intensivos de atención, no así entre las personas retornadas que registran otra condición. Bajo este enfoque, los riesgos están asociados 
con situaciones personales y contextuales negativas que hacen mayor la probabilidad de que las personas desarrollen problemas emocionales, de salud o de otro tipo, como generalmente ocurre entre los migrantes deportados ante el estrés, ansiedad y desgaste asociado al proceso de remoción o la llamada "salida voluntaria".

Además del riesgo, el principio de necesidad tiene como finalidad identificar factores de riesgo estáticos (asignan vulnerabilidad, pero no cambian) y dinámicos (son susceptibles de cambio), que se constituyen como objetos de intervención para la reintegración. Por último, el principio de capacidad de respuesta parte de establecer que existen características cognitivo-conductuales y de personalidad que tienen influencia en la disposición a responder ante los tipos de atención.

Finalmente, el enfoque de redes e intervención comunitaria postula la generación de capital social del migrante en retorno, debido a la vulnerabilidad relacional con respecto a distintas redes sociales, lo que hace por ejemplo que la nula incorporación a sectores estratégicos recaiga en el acceso a empleos con bajos ingresos, limitada estabilidad, sin asistencia y seguridad social, en virtud del desconocimiento sobre los apoyos o ayudas sociales y gubernamentales.

Desde una mirada práctica, encontramos que este modelo recupera diversos elementos conceptuales de los establecidos anteriormente, en la medida en que prioriza a la persona migrante como sujeto de derechos, al tiempo que recupera la dualidad estructura-agencia, en tanto sitúa a la persona en su entorno y contexto inmediato, desde su reconocimiento como agente, con recursos, características y fortalezas que lo dotan de capacidad de respuesta y de acción para su reinserción en los espacios de vida. En otras palabras, reconocemos la importancia de comprender el contexto social de referencia para explicar las acciones y las prácticas a partir de los contextos en los que se gestan y manifiestan (Andrade, 2010).

De ahí la necesidad de identificar los mecanismos que facilitan los procesos de reinserción laboral, así como reconocer el alcance de apoyos gubernamentales entre la población migrante retornada, particularmente al considerar que la vulnerabilidad asociada con el retorno forzado no solo dificulta el acceso a empleos estables, sino que además ha trascendido en un particular énfasis "en el autoempleo, la generación de empresas y negocios que no atiende a las desventajas que provienen del trabajo asalariado y la precariedad laboral" (Masferrer et al., 2017, p. 4).

\section{Dinámica del retorno en el Estado de México}

El abordaje del tema en territorios como el Estado de México reviste importancia ante su posicionamiento como expulsor de migrantes internacionales y de migrantes retornados, dentro de los que ubicamos hombres y mujeres que en muchos de los casos se vieron obligados a regresar, por lo que advertimos que su reinserción a los mercados de trabajo no siempre es sencilla, inmediata y en algunos casos ni siquiera deseada ante las expectativas de regresar a Estados Unidos, sin importar el hecho de haber sido deportados (Jardón \& Baca, 2018).

Para identificar los contextos de retorno y profundizar en el análisis de los procesos de reinserción laboral de la población migrante retornada voluntaria e involuntariamente en el Estado de México tomamos como referente la proporción de viviendas con migrantes que en 2010 se encontraban de regreso en México, en que 
destaca la presencia de municipios con tradición migratoria, ubicados en la región sur de la entidad, como por ejemplo Luvianos, Tejupilco, Coatepec Harinas, Texcaltitlán, Santo Tomás, Malinalco y Ocuilan, principalmente (Consejo Nacional de Población [Conapo], 2012).

Otro acercamiento lo encontramos en la Encuesta Intercensal 2015 (Inegi, 2015), que nos permitió distinguir la asociación entre lugar de retorno y lugar de nacimiento. Entre la población total retornada al Estado de México — que incluye a los nacidos en otras entidades-, el municipio de Ecatepec se constituyó en el principal lugar de asentamiento, seguido por Nezahualcóyotl e Ixtapaluca, así como por otros municipios que en su mayoría corresponden a la Zona Metropolitana de la Ciudad de México (zмсM). No así entre la población retornada que es originaria del Estado de México, entre los que registramos mayor presencia de municipios de tradición migratoria, como son por ejemplo Tejupilco, Tlatlaya y Villa Guerrero (Figura 1).

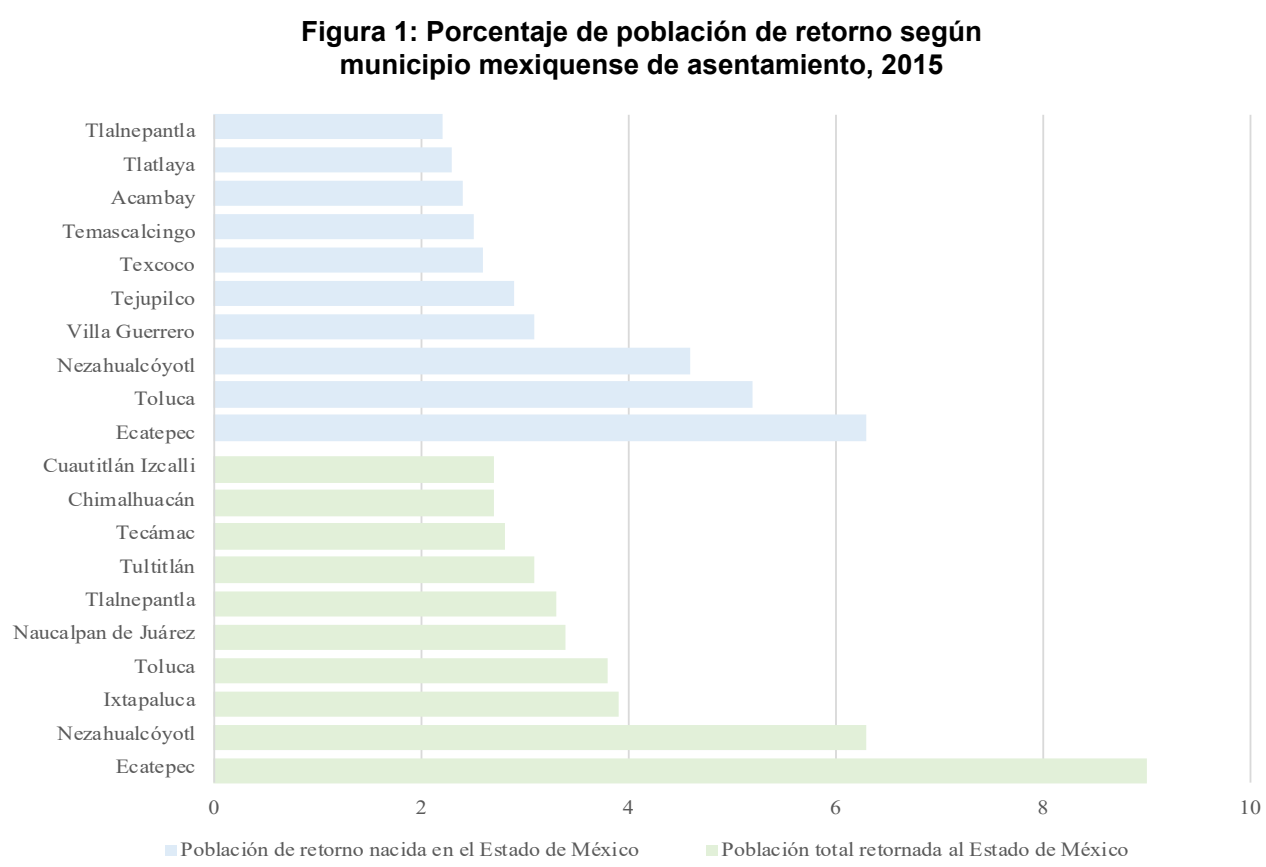

Fuente: elaboración propia con base en la Encuesta Intercensal (Inegi, 2015).

En otras palabras, esta dinámica nos permite explicar que mientras los municipios de tradición migratoria son espacios de recepción de la mayoría de su población originaria que en algún momento emigró. Por el contrario, los contextos urbanos tienden a registrar un mayor dinamismo, o bien, ejercen un mayor nivel de atracción entre la población que no nació en la entidad, lo que se explica por su cercanía con la Ciudad de México y las oportunidades que pueden ofrecer estos espacios, a pesar de la informalidad que adquiere cada vez mayor peso en la zona conurbada del Estado de México.

Finalmente, para aproximarnos en la medida de lo posible a los retornados de manera forzada, nos apoyamos en el flujo de migrantes devueltos por autoridades migratorias de Estados Unidos de la Encuesta sobre Migración en la Frontera Norte 
(Emif Norte). En un mismo sentido, los resultados de esta encuesta arrojan que los municipios mexiquenses con mayor presencia de población retornada a esta entidad fueron Ecatepec, Toluca y Nezahualcóyotl, durante el periodo 2008-2018. Aunque se distingue también la participación de municipios como Ixtapan de la Sal, Tejupilco, Tenancingo y Ocuilan, ubicados en el sur de la entidad mexiquense (Tabla 1).

A partir de esta dinámica, como lo señalamos anteriormente, elegimos el municipio de Nezahualcóyotl por constituirse como un contexto de retorno entre la población nacida en el Estado de México, aunque con una mayor presencia de los que nacieron en otro lugar del país. Lo que nos permite entender la lógica de lo que Masferrer (2014) sostiene, en términos de un retorno que puede ocurrir a otro lugar. Además de que se trata de un municipio cuya movilidad poblacional es dinámica y diversa (Rivera, 2013, p. 64), lo que hace que las redes sociales y familiares de apoyo no siempre estén presentes para facilitar los procesos de retorno forzado y reinserción de esta población.

\begin{tabular}{|c|c|c|c|c|c|c|c|c|c|c|c|}
\hline \multirow{2}{*}{ Municipio } & \multicolumn{11}{|c|}{ Año } \\
\hline & 2008 & 2009 & 2010 & 2011 & 2012 & 2013 & 2014 & 2015 & 2016 & 2017 & 2018 \\
\hline Aculco & & & & & & & 6.2 & 6.8 & & & \\
\hline Calimaya & & & & & & & & & 5.0 & & \\
\hline Chalco & & & & & & & & & & & 21.4 \\
\hline \multicolumn{12}{|l|}{ Cuatitlán Izcalli } \\
\hline Ecatepec & 4.4 & & 3.2 & 5.3 & & 7.4 & & & 5.4 & 10.1 & 5.4 \\
\hline Ixtapan de la Sal & & & & & & & 7.6 & & & & \\
\hline \multicolumn{12}{|l|}{ Morelos } \\
\hline Naucalpan & & 6.6 & & & & & & & & & \\
\hline Nezahualcóyotl & & & 5.2 & & & & & 8.5 & & 10.3 & \\
\hline Ocuilan & & & & & & & & & & 6.0 & \\
\hline Tejupilco & & & & & 7.4 & 6.0 & & & & & \\
\hline Tenancingo & & & & 6.0 & & & & & & & \\
\hline Tlalnepantla & 13.7 & & & & & & & & & & \\
\hline Toluca & 21.5 & 20.9 & 19.6 & 16.7 & 17.7 & 14.5 & 14.6 & 5.8 & 9.7 & & 17.2 \\
\hline Zacazonapan & 7.7 & 4.8 & & & & & & & & & \\
\hline Zinacantepec & & & & & 5.0 & & & & & & \\
\hline $\begin{array}{l}\text { Población total } \\
\text { devuelta de origen } \\
\text { mexiquense }\end{array}$ & 24,302 & 25,532 & 21,270 & 18,593 & 20,684 & 12,635 & 8,175 & 7,610 & 9,168 & 7,323 & 8,534 \\
\hline Proporción respecto & & & & & & & & & & & \\
\hline $\begin{array}{l}\text { del total a nivel } \\
\text { nacional }\end{array}$ & 4.3 & 4.7 & 5.1 & 5.2 & 5.9 & 4.2 & 3.8 & 4.3 & 4.6 & 4.7 & 4.4 \\
\hline
\end{tabular}

Fuente: elaboración propia con base en el flujo de migrantes devueltos (Emif Norte, s. f.).

\section{Perspectivas cruzadas. Entre la reinserción en México y la esperanza de regresar a Estados Unidos}

"Usted puede detener a los pueblos, puede ponerles límites, pero ellos encontrarán otras vías". Esta frase, acuñada por Taylor (2008, en Novosseloff \& Neisse, 2011, p. 28) para hablar de la historia de control y acceso del muro de Berlín, permite de alguna 
manera comprender la eficacia limitada y el efecto contraproducente de los muros que han sido construidos para proteger, separar, rechazar y negar la existencia del otro. Esta ambivalencia se observa en la posibilidad de esquivarlos, que a su vez trasciende en la creación de un sector "sin": "sin papeles, sin estatus" (p. 29).

Esta dinámica que no es ajena a la observada en el muro que separa a México y Estados Unidos ha trascendido en la implementación de diversas acciones de control de la migración irregular, que en algunos casos ha recaído en la muerte de la población que intenta sortearlo, así como en la agrupación de los "sin" entre los muchos que han logrado cruzarlo, o bien, en la deportación de otros tantos cuyo sueño se encuentra ahora del otro lado del muro. Para comprender estas dinámicas, en este apartado exponemos con amplitud el caso de Laura. Para ello, introducimos algunos fragmentos de su narrativa a través de citas textuales de las entrevistas que permitieron reconstruir su proceso migratorio y de reinserción laboral en México.

Para Laura la migración ha sido una constante en su vida, pues, aunque nació en Veracruz, fue registrada, y vivió por 17 años, en Ciudad de México, lugar donde conoció a su exesposo, con el que emigró a Jalisco y vivió por aproximadamente ocho años. Tras su separación, la situación económica de Laura se tornó cada vez más complicada, por lo que sostiene que en ese momento su única opción era irse para Estados Unidos, dejando a sus cuatro hijos de entre uno y nueve años a cargo de su mamá.

Apoyada en sus redes sociales y con la intención de llegar a Chicago, Illinois, Laura intentó cruzar por primera vez por Nuevo Laredo a finales de 2000. Sin embargo, por lo peligroso del cruce, optó por no intentar y regresar "al rancho" para esperar a que le consiguieran otro coyote. En abril de 2001, con ayuda de un conocido de Estados Unidos viajó a Tijuana, ciudad fronteriza desde la que realizó tres intentos, de los que la primera vez señala haber sido devuelta de manera inmediata, mientras que en la segunda ocasión estuvo en un centro de detención por aproximadamente una semana. No obstante estas experiencias, la determinación de Laura, su deseo porque "sus hijos no volvieran a pasar miserias" y el hecho de pensar que "no podía regresar asî" la llevaron a realizar un tercer intento, siendo entonces cuando logró cruzar y llegar a Chicago. En su narrativa Laura expresa lo siguiente:

Mi exesposo es del estado de Jalisco, por eso yo me fui a vivir al estado de Jalisco, en un pequeño pueblito, un ranchito muy chiquito. Por situaciones personales y violencia doméstica me quedé sola con mis cuatro hijos que tenía en ese momento y la situación económica se puso muy difícil para mí. De alguna manera mi única opción en ese momento era irme a Estados Unidos, en estos pequeños ranchitos pues toda la gente prácticamente se va, y la persona que me invitó, que me costeó el coyote estaba en Chicago. Por eso es que yo me fui a Chicago, porque depende el rancho es como se van yendo y nos vamos acomodando en Estados Unidos. Hice un primer intento por Laredo, pero era muy peligroso, me regreso al rancho y espero a conseguir que me consiguieran otro coyote y ya es como me voy a Tijuana. Entonces voy a Tijuana, hago un primer intento y me regresan, intento días después otra vez, me detienen, estoy en un centro de detención como una semana, me sacan, y yo ahí me decidí que no iba a volver pues no quería que mis hijos volvieran a pasar miserias de nada, no podía regresar así, tenía que intentarlo. En el tercer intento logro pasar y es así como llego a Chicago. 
Al estar en esa ciudad, para Laura como para muchos migrantes, el plan era quedarse solo uno o dos años para juntar dinero y regresarse. Sin embargo, su estancia en Estados Unidos se prolongó por aproximadamente 15 años, tiempo durante el que inició otra relación y tuvo otros dos hijos. Durante su permanencia en Chicago la vida de Laura fue "normal como la de cualquier migrante". A tan solo un mes de haber llegado empezó a trabajar en Unique, una cadena de tiendas de segunda mano, empleo en el que se mantuvo por más de 10 años realizando actividades diversas.

A pesar de que Laura advierte que su vida fue como la de cualquier migrante, reconoce también que su historia cambia cuando en 2010 empieza a estudiar para obtener el Diploma de Educación General (General Education Development, o GED), equivalente a la preparatoria, además de que ya tenía un mayor dominio del idioma inglés. Estos recursos le permitieron iniciar su participación como activista, particularmente cuando en 2011 la tienda en la que se encontraba trabajando fue vendida y los nuevos dueños empezaron con el recorte de personal, conservando a los de mayor antigüedad, con conocimiento sobre la administración y manejo de la tienda, y con habilidad para capacitar a los nuevos empleados.

La visibilidad de Laura como migrante no documentada perteneciente a ese grupo de los "sin" se hizo nítida con los alcances del programa de verificación electrónica E-Verify, cuyo principal antecedente está en la Ley de Reforma y Control de la Inmigración de 1986 (IRCA, por sus siglas en inglés), dicha ley requirió que los empleadores examinaran la documentación de cada trabajador recién contratado para probar su identidad y elegibilidad para laborar en Estados Unidos, además que desembocó en la creación del Formulario I-9, Verificación de Elegibilidad de Empleo. Posteriormente, con la Ley de Reforma de Inmigración Ilegal y Ley de Responsabilidad del Inmigrante de 1996 (IIrIRa, por sus siglas en inglés) se promueve el Programa Piloto Básico que en 2007 cambia su nombre a E-Verify, que con el tiempo adquirió nuevas funciones y herramientas para validar la información (E-Verify, 2018).

En una actualización realizada en 2011 se encuentra el denominado E-Verify Self Check que permitió que los empleadores de Laura anunciaran que al año siguiente habría una verificación de documentos. Dicha advertencia, pero también la prohibición de hablar español —sustentada en iniciativas como English only- y otras prácticas de hostigamiento y presión hacia el personal en su mayoría femenino, de nacionalidad mexicana y estatus no documentado, hacen que Laura comenzara a capacitarse a través de la organización Arise Chicago, donde cursa un taller sobre derechos laborales e inicia una campaña de sindicalización en su tienda, con el fin de procurar la protección de sus derechos y defender el hecho de que las personas con antigüedad laboral no tenían que ser partícipes de la revisión de papeles.

No obstante, la campaña promovida por Laura no logró su objetivo, probablemente como resultado del declive del sindicalismo y de la negociación colectiva en Estados Unidos (Zepeda, 2016; La Botz, 2018), pues en este caso únicamente podían votar los trabajadores con mayor antigüedad. Fue así como en enero de 2013 inició la solicitud de documentos para actualizar la información de los trabajadores, que en su mayoría eran no documentados. Este hecho provocó que Laura y compañeros no regresaran a laborar a la tienda porque los iban "a atracar". Como resultado, entablan y ganan la demanda que interpusieron ante la Junta Nacional de Relaciones Laborales. Aunque ello fue simbólico, señala Laura, porque ganaron su derecho a regresar al trabajo, pero los empleadores tenían también el derecho de revisar sus documentos. 
A un mes de que Laura se quedara sin empleo, logra conseguir otro trabajo a través de una agencia de colocación, aunque reconoce que no fue fácil por el programa E-Verify. Sin embargo, conocedora ya de sus derechos que le permiten tener otra perspectiva y reconocer situaciones de abuso laboral, después de seis meses decide dejar de trabajar, vivir de sus ahorros, estudiar y participar de manera más activa como voluntaria en organizaciones como Centro Romero Chicago, donde impartía cursos de alfabetización, pues la experiencia con sus compañeras de trabajo de la tienda Unique le permitió sensibilizarse con respecto a los abusos y las mayores dificultades que experimentan las personas migrantes cuando no saben leer y escribir.

Mujeres Latinas en Acción fue otra de las organizaciones en la que Laura empezó a involucrarse, primero por consejería dada la situación de violencia psicológica que vivía por parte de su esposo, y después como asistente a los cursos que imparte dicha organización para capacitar a las mujeres para "emprender algo a partir de cualquier cosa que sepan hacer". La gratuidad de los servicios proporcionados por la organización le permiten no solo realizar diversos cursos sobre liderazgo, emprendimiento y computación, sino convertirse en instructora de los talleres sobre derechos laborales en Arise Chicago, organización en la que inició sus conocimientos sobre el tema.

El involucramiento que Laura tuvo en Arise Chicago la llevó a convertirse en la coordinadora de educación en 2015, y quedarse a cargo de los talleres sobre derecho laboral, por lo que continuó con su capacitación con la agencia responsable de la Administración de Seguridad y Salud Ocupacional del Departamento de Trabajo de Estados Unidos (osha, por sus siglas en inglés). Con ello, Laura dejó de ser voluntaria en la organización y su labor en esta se constituyó en el que fuera su último trabajo, por un tiempo de casi cuatro años.

Aunque por el hecho de no tener documentos su pago lo recibía bajo la modalidad de beca, para Laura "era el sueño" porque pasó de percibir el mínimo por hora durante los 10 años que trabajó en Unique, a 21 dólares la hora en Arise Chicago. Además "de que estaba haciendo otras cosas que nunca imaginó" pues en el que fuera su lugar de trabajo le pagaban cursos sobre organización comunitaria y sindicalismo en la Universidad de Illinois, y estuvo a cargo de la semana laboral dentro del consulado de México en Chicago.

A la par de que Laura consideraba estar viviendo "el sueño" laboralmente, dice también que su vida personal "estaba tremenda y se sentía acorralada porque quería librarse de esa relación, pero el no tener documentos no facilitaba eso". Surge entonces la idea de que la organización la ayudara pidiéndola como su trabajadora. Para ello, como puede leerse en la narrativa sobre la trayectoria migratoria y la deportación de nuestra informante. Laura tenía que regresar a México a iniciar su proceso, que no ocurrió ya que en el aeropuerto fue abordada por migración y deportada en junio de 2016 con una penalidad de 20 años.

Platicando con la organización, pues salió la idea de que ellos me ayudaran y me pidieran como su trabajadora. Siempre digo que me tragué también el discurso de que solamente se deporta a la gente que comete algunos errores o crímenes, y dije, bueno, yo no tengo ningún delito, siempre pagué mis impuestos, no tengo ni un ticket de tránsito siquiera, dije, tengo más cosas a mi favor que en mi contra. Lo que yo no recordaba en ese momento, lo que yo había olvidado son las dos agarradas en la frontera. Yo necesitaba salir de Estados Unidos para iniciar el proceso, sabía que era arriesgado. Entonces, yo 
compré en junio de 2016 un boleto para venir a México para iniciar ese proceso justamente. Pues llegué al aeropuerto normal, documenté, paso seguridad nacional y ya para abordar estaba migración en la puerta del avión, no era una redada, ni estaban pidiendo sus documentos a nadie, solamente estaban ahí por mí. Entonces, ellos cuando me vieron se acercan y me piden mis documentos, les enseño mi pasaporte y mi pase de abordar y me dicen que los tengo que acompañar, ya caminando hacia su oficina que tienen ahí adentro del aeropuerto el agente me preguntó ¿has tenido deportaciones? y le dije no y me empezó a preguntar más cosas y le dije yo no te voy a responder ya nada, yo no tengo porqué responderte, ya llegando lo que sí ya no me pude negar es que revisaran mis huellas. Al revisar mis huellas obvio salió el récord de las dos agarradas y que esas dos agarradas no son agarradas, son deportaciones. Digamos que fue la excusa que usaron para darle un respaldo legal a lo que hicieron en mi caso, porque el trasfondo de mi deportación es más bien el activismo y pienso que cuando empecé la campaña de sindicalización la misma compañía me puso el dedo, pero Chicago es ciudad santuario, no te pueden deportar tan fácilmente, pero de alguna manera se dieron las circunstancias perfectas, en zona federal no entra lo de ciudad santuario. Entonces pues me detienen ahí, yo tontamente firmé, me bloqueé, no sé, tantas cosas pasaron por mi mente en ese momento, tontamente firmé que estaba aceptando la deportación, me regresan al avión y ya iba con la deportación y la penalidad de 20 años para ya no regresar a Estados Unidos. Ahí es donde cambia todo completamente y llegando aquí obvio pues todo fue ¿ahora qué voy a hacer? o sea yo venía con la idea de hacer esto, tenía la idea de que al fin iba a arreglar mis papeles y pues lo más doloroso que pensaba que iba a volver a ver a mis hijos. Entonces llegar sí fue bastante difícil, digo, en mi caso los dos primeros años fueron los más difíciles, sigue siendo difícil, pero ahorita ya estoy en un proceso, tengo que asimilar las cosas, tengo que aceptar las cosas y no puede acabarse mi vida por una deportación.

Lo anterior ocurre a pesar de encontrarse en una ciudad santuario, una ciudad de bienvenida y que trabaja para mantener sus políticas y programas para la incorporación de los migrantes sin importar su estatus migratorio (Cruz, 2019). El sustento legal de la deportación de Laura se tiene en la Ley de 1996, con las provisiones que establecen penas civiles a las entradas ilegales y la autorización de tomar las huellas de los migrantes capturados, por lo que, al ser arrestados y enviados a un centro de detención, como ocurrió con Laura, los migrantes quedan con antecedentes policiales (Castañeda, 2012, p. 309).

La experiencia de Laura pone de manifiesto que las políticas de control migratorio se han enfocado en expulsar a la población establecida de manera permanente en Estados Unidos, con deportaciones que para estos migrantes significan más que la pérdida de un ingreso en dólares, pues regularmente conlleva la separación de familias con estatus migratorios mixtos (Peña, 2017).

Estar de regreso en México, si bien no ha sido fácil para Laura, también reconoce que su vida no puede acabarse por una deportación. Motivo por el que ha buscado reinsertarse en el municipio de Nezahualcóyotl, Estado de México. Lugar en el que vivía antes de haber migrado a Jalisco y que "le abrió las puertas" a su regreso en 2016, aunque también muchos de sus vínculos los tiene en la Ciudad de México, pues 
después de haber sido deportada fue en las instituciones de esta ciudad donde empezó a buscar apoyos, y logró obtener la ayuda del Programa Seguro de Desempleo de la Secretaría del Trabajo y Fomento al Empleo (Styfe), con un monto de $\$ 2,100$ pesos mensuales durante seis meses.

En diciembre de 2016, como puede leerse en su narrativa, Laura y otros beneficiarios de dicho programa son convocados para formalizar la recepción de su apoyo. En el evento al que acudió, además de percatarse de que no está sola pues son varios los deportados, fue invitada a ser parte del presídium, ya que su participación como activista en Chicago le permitió conocer a diversos servidores públicos, dos de los cuales se encontraban presentes en dicho evento.

Para iniciar algo tenía que permanecer en una ciudad estratégica como la Ciudad de México, por eso me quedé aquí y empecé a ver qué más había y empecé a saber de los programas que en ese momento existían para atender a nuestra comunidad. Fui y apliqué a uno de ellos que es el seguro de desempleo de la Secretaría del Trabajo local, es el Styfe, entonces apliqué y sí me hablaron y me citaron el día 6 de diciembre de ese mismo año, supuestamente para recibir el beneficio, y ya fui, porque como era para la foto citaron a mucha gente deportada y retornada. Entonces fue ahí cuando dije no pues no estoy sola, somos muchos. Cuando llegué estaba un político de Chicago, el cual yo conocía, pero por el mismo trabajo que yo hacía con la organización, y también estaba ahí la que era en ese momento la secretaria del trabajo, la señora Amalia García, que también la conocí desde Chicago, y cuando me ve acá me dice ¿qué haces aquí? y ya le conté, y me dice, lo siento mucho Laura, y me invitan a pasar al presídium, entonces ella hace una mención sobre mí y dice que lamenta mucho que esté yo ahí porque sabe del trabajo que yo hacía allá, pero que bienvenida a México. Eso le llamó la atención a algunos de los compañeros y cuando se acabó el evento me preguntaron, ¿ioye, pero entonces qué hacías tú allá? y nos quedamos platicando y estuvimos hablando de los temas de lo que nos pasó en México, de lo que habíamos hecho allá y vi muchas cosas similares, las dificultades para conseguirnos nuestros documentos de identidad que es una de las primeras necesidades para adaptarse, de que estábamos separados de nuestros hijos. Entonces ahí mismo les dije: a ver, yo traigo esta experiencia de Chicago, podemos organizarnos, podemos hacer un grupo y visibilizar lo que está pasando aquí en México, tomé teléfonos, nombres y la siguiente semana les estuve llamando y nuestra primera reunión fue allá afuera del museo Franz Mayer el 16 de diciembre de 2016, y a partir de ahí nace esto.

Este evento, pero particularmente el capital humano y político, le permitieron vincularse con otros migrantes, en su mayoría del Estado de México, con los que forma el denominado Colectivo Deportados Unidos en la Lucha (DUL). Como grupo organizado empezaron a vender dulces en diferentes puntos de la Ciudad de México, con el fin de obtener ingresos, pero también con el propósito de sensibilizar a la gente respecto del constructo del migrante deportado como criminal, asesino, violador, que hizo y hace que Laura reciba mensajes de odio en los que se refieren a ellos como "huevones antipatria, ratas" y otras expresiones que en su momento hicieron que a ella misma le diera vergüenza decir que era deportada. Toda vez que el retorno por causales de deportación redirecciona la migración forzada hacia un espiral de mayor 
degradación social, en el que las personas sufren criminalización y el retorno simboliza un segundo destierro (Márquez, 2013, p. 160).

De manera alterna a estas actividades, Laura tenía su trabajo como maestra de apoyo de inglés a nivel preescolar y primaria en una escuela particular de Nezahualcóyotl. Este empleo lo obtuvo con relativa facilidad tanto por sus conocimientos como por su capacidad de gestión, aunque se trató de un trabajo que no cumplía sus expectativas en términos del salario y de las actividades realizadas, además de la "incomodidad" asociada con que le pidieran que se cubriera sus tatuajes, "signo de criminalidad y riesgo para la ciudadanía” (Albicker \& Velasco, 2016). Todo ello incidió en su decisión de dejar ese trabajo para dedicarse a sus actividades en el Colectivo Dul, que Laura considera que es "su causa, su vida y su familia".

Reiniciar su vida, reinsertarse ella misma y apoyar a otros "compas" a través de esta "red solidaria de amigos" son algunos de los significados que Laura encuentra en el colectivo. Dedicada a esta actividad, junto con sus "compas" pasa de vender dulces a vender playeras con motivos y mensajes vinculados con la migración. Así fue como partiendo de los conocimientos adquiridos en los cursos sobre emprendimiento y liderazgo que realizó en diversas organizaciones de Chicago "tuvo los cimientos" para presentar ante la Styfe una iniciativa de ocupación por cuenta propia, como parte de las acciones que esta dependencia proporcionó en junio de 2017 para desarrollar proyectos productivos.

Para ello, se constituye el proyecto Deportados Brand, en colaboración con otras cuatro personas migrantes, y obtienen un presupuesto de $\$ 115,141$ pesos para adquirir maquinaria e iniciarse en el giro de la serigrafía (Secretaría de Trabajo y Fomento al Empleo, 2018). Desde entonces y a la fecha, Laura se desempeña laboralmente en su taller, que en su opinión "se ha convertido de un proyecto productivo a un emprendimiento, donde están autoempleados y esperan pasar a empresarios y consolidarse más" con productos que ya no solo son comercializados en México, sino que envían a diversos puntos de Estados Unidos, particularmente a Chicago.

A su vez, los productos que ofrece Deportados Brand se han venido diversificando, lo que da cuenta de la capacidad de inventiva, arrojo y adaptación de Laura y Gustavo, actuales administradores del negocio. Entre estos, además de las playeras, comercializan bolsas, tazas, gorras, stickers, ropa, y recientemente se encuentran iniciando una nueva etapa como taller de costura que coincide con las afectaciones en su economía por la crisis asociada con la emergencia sanitaria por Covid-19, que puso nuevamente a prueba su capacidad de reinventarse y de ajustarse elaborando cubrebocas, con el fin de ofrecer una respuesta también a la demanda del mercado.

Entre otras estrategias, Laura está convencida de la necesidad de establecer vínculos para ampliar los alcances de su negocio, por lo que se puso en contacto con el presidente municipal de Nezahualcóyotl para que les dieran apertura para vender sus productos. Hoy en día, además de ser invitados a determinados eventos en los que les permiten su venta, han diseñado "la línea Neza" con modelos como "NezaYork" y "Minezota" a través de los que buscan "poner el orgullo de estar en Neza" para resaltar aspectos positivos y cambiar la imagen que se tiene del lugar, además de convivir y aportar a la gente del municipio. Al respecto, Laura señala lo siguiente:

Ya tenemos también el acercamiento con el municipio de Neza, nos dan apertura para vender de vez en cuando, nos invitan a sus eventos también para vender de vez en cuando. A raíz de todo eso surgió nuestra línea de Neza, 
porque mucha gente también decía... jay! Neza no, no querían ni venir, dije no, pues aquí estamos, y luego mejor la colonia que nos ha abierto las puertas. La realidad de muchos deportados es que estamos en el Estado de México, digo, los más que están son de Ecatepec y Nezahualcóyotl. Entonces acá en Neza hemos hecho también el acercamiento con el presidente municipal, en el ayuntamiento, y en cuestión de Deportados Brand tenemos nuestra propia línea de diseños en Nezahualcóyotl, pues porque ahora estamos aquí, y también resaltar un poquito lo que se está haciendo aquí en Neza, hemos conocido gente que está aquí, haciendo cosas chidas, entonces estamos tratando de abrir esos espacios para poder hacer nuestra misión de la gente deportada, de los productos o de las cosas que están desarrollando.

En tal sentido, Deportados Brand es su recurso para la generación de ingresos, mientras que el Colectivo dul se ha constituido en la plataforma que le permite dar continuidad a su papel como activista y promotora de diversas acciones de incidencia para facilitar la reinserción de los migrantes deportados, así como para crear una red de emprendedores deportados, consumir sus productos y darse trabajo entre ellos mismos. Al mismo tiempo, se trata de un espacio que le ha permitido vivir, experimentar y llevar su propio proceso de deportación y reinserción en México, pues a pesar de sus muchos esfuerzos reconoce que todavía "le agarra la nostalgia y se deprime" y alberga la esperanza de que algún día las leyes de Estados Unidos cambien para que haya una libre movilidad.

$\mathrm{Al}$ respecto, señala que esa "obsesión de regresar a Estados Unidos" es la que no hace posible y dificulta la reinserción en México, por lo que "cambiar la mirada del norte al sur" es fundamental, como ocurrió con ella, cuando a principios de 2019 "le pusieron los pies en la tierra" sobre su situación migratoria. Aunque sabe que la esperanza de regresar "siempre va a estar ahí", en su narrativa sostiene que está tratando de dejar de mirar al norte y voltear al sur, apoyándose de las redes que está construyendo con los grupos de deportados.

En esta constante lucha que involucra sentimientos y emociones contradictorias, Laura se ha convertido en ejemplo para otros migrantes que en una situación semejante a la de ella consideran que también pueden salir adelante en México. Por ello, a través del colectivo, según lo advierte en su narrativa, busca concientizar y motivar a los "compas" a que cambien la mirada y adquieran las herramientas para reinsertarse en México, bajo un discurso que resalta la importancia de capacitarse y de adquirir conocimientos que también les pueden ser útiles en caso de que ocurra el regreso a Estados Unidos.

Una de las partes fundamentales para lograr una reinserción exitosa es quitarte la mirada para el norte, mientras tú sigas obsesionado con regresar hacia Estados Unidos va a ser muy difícil, pero si se los platica a los compas alguien más que no ha sufrido la deportación, pues no lo van a creer, pero en este caso, los compas nos ven que nosotros mismos lo estamos haciendo, se inspiran y eso es lo que estamos haciendo, por eso es que ya hay más emprendimientos. Yo no quito, no está fuera de mi mente que las cosas cambien y haya una libre movilidad, que las leyes cambien en Estados Unidos, pero no es mi obsesión, ni en lo que gasto mi vida, porque mejor acá hago, construyo mi vida, al final de cuentas lo que les hago ver a los compas es que, si tienes tantas ganas de regresar, al final del día estos son elementos para regresar, porque ya no 
es lo mismo. Entonces, en vez de estar llorando, victimizándote, por qué no aprovechas ese tiempo y te pones a estudiar, te pones a trabajar, te pones a hacer eso y reúnes elementos que si en un día puedes hasta para calificar para una visa de turista, te vas.

La reinserción laboral y las estrategias que Laura ha emprendido a nivel individual y colectivo han ocurrido sin que necesariamente su plan fuera prepararse para una deportación y quedarse en México, pues, así como lo fue en su caso, "la gente trata de pensar en quedarse y no en regresar, la gente ni piensa en eso, ni quiere saber nada de la deportación, lo que quieren es poder arreglar sus papeles".

Es por ello —explica— que iniciativas como "el plan es hacer un plan" no son del interés de la población migrante, pues en todo caso se requiere primero "comprender su realidad, hablar su lenguaje, entender las dos realidades" para explicar ¿por qué después de haber vivido en otro lugar lo que buscan es permanecer allí? ¿Y por qué después de haber sido deportados no siempre buscan establecerse sino regresar al que se ha constituido en su hogar?

Al respecto, los relatos de Laura, como lo señalamos anteriormente, permiten advertir que la obsesión y/o esperanza siempre viva por regresar a Estados Unidos de muchos de estos migrantes tiene que ver con la construcción de lo que es su hogar, su casa, su vida, en la medida en que la misma Laura, quien considera haber cambiado su mirada hacia el sur y haberse establecido en Nezahualcóyotl, "se resistía" en amueblar su departamento, pues no le encontraba sentido al pensar que iba a regresar a Estados Unidos, ya que su casa está allá y allá están sus cosas. Así pues, la construcción de lo que es la casa y el hogar, que a la fecha para ella sigue estando en Chicago, es uno de los principales factores en los que debemos pensar para reconocer las necesidades y acciones de la población migrante deportada. En otras palabras, reflexionar sobre los procesos de reinserción de esta población nos requiere avanzar en la comprensión de otros aspectos que van más allá de lo laboral, en tanto se conjugan con un cúmulo de emociones y sentimientos que transcienden también en los procesos de aceptación y/o negación sobre el retorno y la reinserción.

Mi concepto de lo que yo entiendo de hogar está en Chicago y eso es mucho de lo que a veces la gente no entiende, que es mucho más, es tu vida, es tu arraigo y no es fácil dejar donde has construido, yo lo he dicho, para mí ha sido tan difícil, que ahorita yo ya empiezo a amueblar mi casa, mi departamento donde estoy, yo me negaba a amueblarlo o a comprar cosas, ¿para qué si yo me voy a regresar? tarde o temprano me voy a regresar, ¿para qué compro eso? ¿para qué pongo el otro? si mi casa está allá y allá están mis cosas, y me ha costado trabajo porque yo sigo viendo mi casa allá, o sea, imagínate que tu taza favorita, que tus plantas, tu almohada, todo lo que construye tu vida más allá del trabajo, más allá de cualquier otra cosa, más allá de los dólares, toda tu vida está allá, ¿cómo lo cambias?, ¿cómo le haces a la gente entender que un día las cosas van a cambiar? que van a tener que renunciar, que tienen que prepararse para renunciar a eso. No es fácil, y yo creo que esa es la negación.

A modo de cierre, puede decirse que el caso de Laura se inscribe dentro del prototipo de reinserción con establecimiento que Rivera (2013, p. 72) propone para referirse a la población migrante cuya experiencia personal se interseca con las condiciones históricas como inmigrante no documentada en Estados Unidos. Esto 
es, las detenciones en sus intentos de cruce, la situación de violencia psicológica por su pareja en Estados Unidos, la falta de documentos para poder independizarse, su participación como activista en materia laboral en Chicago, el desarrollo de un proyecto productivo que trascendió en emprendimiento en México, la organización del colectivo Dul para facilitar la reinserción de sus "compas" y la decisión de cambiar la mirada del norte al sur.

Se trata de un conjunto de acciones que ponen de manifiesto su papel como agente en la promoción de su propio proceso de reintegración social, laboral y cultural, indistintamente de los muchos conflictos que pudo haberle generado la criminalización y estigmatización de la figura del migrante deportado, así como de los choques e impactos para adaptarse después de haber estado un amplio periodo fuera de México, con altas y bajas que responden a la todavía construcción y asociación de su hogar en Chicago.

Es claro también que el proceso de reinserción de Laura fue facilitado por la disposición de diversos capitales, que como recurso acumulado durante su trayectoria migratoria le permitieron promover un conjunto de diversas estrategias tanto individuales, como colectivas en contextos como el Estado de México y la Ciudad de México, donde dispuso de las condiciones para reconvertir dichos capitales. Situación que en materia laboral, por ejemplo, hizo de su regreso, aunque forzado, un retorno productivo, además de propiciar el uso de sus habilidades, conocimientos y activos adquiridos en Estados Unidos.

\section{Conclusiones}

La historia y experiencia de Laura, corresponde a un caso, que ella misma señala, tiene diversas similitudes en torno a las necesidades individuales, familiares e institucionales asociadas con el regreso forzado. A pesar de estas semejanzas, ha sido de nuestro interés enfatizar en la atipicidad de su caso, por lo que el abordaje de su narrativa en el desarrollo de este artículo permite poner en evidencia que la agencia, iniciativa y acumulación de capitales trasciende en las formas de negociar y gestionar una reinserción exitosa en contextos que, aunque se perciben ajenos, son estratégicos para la reconversión y uso de dichos capitales.

Si bien en este artículo hemos enfatizado en los componentes laborales, a partir de la narrativa de nuestra informante, reconocemos la interrelación con otros aspectos sociales, culturales, institucionales y políticos que precisan la comprensión de la reinserción desde una mirada integral. Esto, a su vez, implica reconocer la complejidad que dichos procesos adquieren a partir de los perfiles heterogéneos de la población migrante, de la experiencia vivida en Estados Unidos, así como de las condiciones, temporalidad y contexto en el que está ocurriendo el retorno.

Con ello buscamos advertir que aun cuando la liquidez y solvencia económica sea una de las primeras necesidades de las personas migrantes deportadas, lo cierto es que no siempre estarán preparadas para reincorporarse de manera inmediata, pues cada persona experimentará y vivirá su proceso de deportación y reinserción de diferente manera. A manera de ejemplo, en contraste con el caso de Laura, otras entrevistas realizadas con la población deportada en contextos rurales del Estado de México ponen de manifiesto situaciones no solo de desconocimiento de los apoyos 
gubernamentales disponibles, sino también de falta de empleo, habilidades y activos que no pueden ser utilizados, así como otros recursos personales de los que no se valen para contrarrestar las dificultades, sino para recrear escenarios desde los que perciben un México en el que no pueden salir adelante.

En tal sentido, la reinserción de esta población se constituye en un reto del que deben participar tanto los gobiernos como la población migrante retornada y deportada, las organizaciones de la sociedad civil y la sociedad en su conjunto, lo que supone entonces que la integración involucra la suma de esfuerzos colectivos, más que individuales. Como ha sido documentado en el caso aquí analizado, la reinserción de Laura en mucho tuvo y tiene que ver con la determinación y voluntad para vincularse con el sector público, la propia comunidad de "compas" deportados y la sociedad en general para establecer rutas orientadas a facilitar la experiencia de la deportación y la reintegración en México, para luchar por un sueño que de momento descansa todavía del otro lado del muro, aunque no por ello ha dejado de emprender diversas acciones para contribuir y apoyar a otros que han vivido la deportación.

Para terminar, consideramos importante señalar que el caso de Laura aporta a los estudios sobre deportación y reinserción laboral en tanto destaca el alcance que la agencia, la disposición de capitales y la capacidad para reconvertirlos pueden tener para asegurar los procesos de reintegración individual y colectiva de esta población. De ahí la necesidad de comprender las necesidades y reconocer la heterogeneidad en los perfiles de los migrantes deportados, pues de ello depende el diseño y la implementación de iniciativas y mecanismos eficientes para facilitar dichos procesos entre esta población que se encuentra en una constante negociación entre reinsertarse en México y regresar a Estados Unidos.

\section{Referencias}

Alanís, F. (2005). Regreso a casa: la repatriación de mexicanos en Estados Unidos durante la gran depresión. El caso de San Luis Potosí, 1929-1934. Estudios de Historia Moderna y Contemporánea, (29), 119-148. http://www.scielo.org.mx/scielo. php?script=sci_arttext\&pid=S0185-26202005000100119

Albicker, S. \& Velasco, L. (2016). Deportación y estigma en la frontera México-Estados Unidos: atrapados en Tijuana. Norteamérica, 11(1), 99-129. https://doi. org/10.20999/nam.2016.a004

Andrade, L. (2010). Revisitando el oficio del sociólogo. Notas sobre el habitus del investigador social. Cinta Moebio, (39), 153-169. https://scielo.conicyt.cl/scielo. php?script=sci_arttext\&pid=S0717-554X2010000300003

Anguiano-Téllez, M, Cruz-Piñeiro, R. \& Garbey-Burey, R. (2013). Migración internacional de retorno: trayectoria y reinserción laboral de emigrantes veracruzanos. Papeles de Población, 19(77), 115-147. http://www.redalyc.org/articulo. oa?id=11228794005

Beltrán, E. (2019). Frente al panorama del retorno: experiencias de personas nacionales deportadas de Estados Unidos a México, vía Ciudad Juárez (tesis de maestría). Universidad Autónoma de San Luis Potosí. https://ninive.uaslp.mx/ xmlui/handle/i/5653 
Castañeda, A. (2012). La violencia de la ley: la legislación migratoria y el proceso de deportación. En M. D. París (Coord.), Migrantes, desplazados, braceros y deportados. Experiencias migratorias y prácticas políticas (pp. 299-331). El Colegio de la Frontera Norte.

Castles, S. (2012). Understanding the relationship between methodology and methods. En C. Vargas-Silva (Ed.), Handbook of research methods in migration (pp. 7-25). Edward Elgar Publishing, Inc.

Consejo Nacional de Población (Conapo). (2012). Índice de intensidad migratoria por entidad federativa y municipio 2010. Autor.

Cruz, E. (2019). El espectro de las ciudades santuario en Estados Unidos: Los contrastes en la génesis y las prácticas de las políticas locales proinmigrantes. Estudios Fronterizos, 20(29), 1-22. http://dx.doi.org/10.21670/ref.1908029

Durand,J. (2013). Nueva fase migratoria. Papeles depoblación, 19(77), 83-113. http:/ / www. scielo.org.mx/scielo.php?script=sci_arttext\&pid=S1405-74252013000300007

El Colegio de Tlaxcala A. C. \& Gobierno del Estado de Tlaxcala. (2017). Evaluación específica de desempeño. Fondo de Apoyo a Migrantes del Estado de Tlaxcala, 2016. Ramo 23 Previsiones Salariales y Económicas. Autores. http://evaluacion.tlaxcala.gob. $\mathrm{mx}$ /images/stories/documentos/evalua/evaluaciones/ef16/fondo_mig_ef16. pdf

Encuesta sobre Migración en la Frontera Norte (Emif Norte). (s. f.). El Colegio de la Frontera Norte.

E-Verify. (2018). Historia e hitos de E-Verify. Resumen cronológico de los hitos del Programa E-Verify: 1986. DHs \& Uscis https://www.e-verify.gov/es/acerca-de-everify/historia-e-hitos-de-e-verify\#y1986

Flyvbjerg, B. (2004). Cinco malentendidos acerca de la investigación mediante los estudios de caso. Reis, 106(4), 33-62. http://www.reis.cis.es/REIS/PDF/ REIS_106_041167998142322.pdf

Gullahorn, J. \& Gullahorn, J. (2013). Extension of the U-Curve Hypothesis. https:/ /prezi. $\mathrm{com} / \mathrm{v}$-uazttao6lh/extension-of-the-u-curve-hypothesis/

Gutiérrez, A. (2004). Acerca del capital social como herramienta de análisis. Reflexiones teóricas en torno a un análisis de caso. IV Jornadas de Sociología. Facultad de Ciencias Sociales, Universidad de Buenos Aires. http://cdsa.aacademica. org/000-045/10.pdf

Instituto Nacional de Estadística y Geografía (Inegi). (2015). Encuesta Intercensal 2015.

Jardón, A., Ronzón, Z. \& Murguía, V. (2019). Procesos de reinserción laboral de la población migrante devuelta de Estados Unidos a México. La situación demográfica de México, 1, 215-230. https://www.gob.mx/cms/uploads/attachment/ file/528759/LSDM_2019_OK_27ENE2020_LOW.pdf

Jardón, A. \& Baca, N. (2018). Dinámica contemporánea del retorno migratorio: estudio de caso en comunidades del sur del Estado de México. Século XXI. Revista de Ciências Sociais, 8(1), 233-265. http://ri.uaemex.mx/handle/20.500.11799/95257

La Botz, D. (2018). EEUU: Trump y el movimiento sindical, más allá de los daños inmediatos. Sin Permiso. https://www.sinpermiso.info/textos/eeuu-trump-y-elmovimiento-sindical-mas-alla-de-los-danos-inmediatos

Márquez, H. (2013). El redoble de la migración forzada: inseguridad, criminalización y destierro. Migración y Desarrollo, 11(21), 159-175. http://www.scielo.org.mx/ scielo.php?script=sci_arttext\&pid=S1870-75992013000200007 
Masferrer, C. (2014). De regreso a otro lugar. La relación entre migración interna y la migración de retorno en 2005. En F. Lozano \& M. Valdivia (Comps.), Análisis espacial de las remesas, la migración de retorno y crecimiento regional en México (pp. 175-238). Plaza y Valdés.

Masferrer, C, Sánchez, L. \& Rodríguez, M. (2017). Condiciones laborales de los migrantes de retorno de Estados Unidos. Apuntes para la equidad, (2), 1-4. https:// migdep.colmex.mx/actividades/images/sem-politicas-laborales-retornados.pdf

Massey, D., Durand, J. \& Malone, N. (2009). Detrás de la trama. Políticas migratorias entre México y Estados Unidos. Cámara de diputados, Lx Legislatura, Universidad Autónoma de Zacatecas, M. Ángel Porrúa.

Massey, D, Pren, K. \& Durand, J. (2009). Nuevos escenarios de la migración México-Estados Unidos. Las consecuencias de la guerra antiinmigrante. Papeles de Población, 15(61), 101-128. https://rppoblacion.uaemex.mx/article/view/8534

Moctezuma, M. (2008). El migrante colectivo transnacional: senda que avanza y reflexión que se estanca. Sociológica, 23(66), 93-119. http://www.scielo.org.mx/ scielo.php?script=sci_arttext\&pid=S0187-01732008000100005

Novosseloff, A. \& Neisse, F. (2011). Muros entre los hombres. El Colegio de la Frontera Norte.

Organización Internacional para las Migraciones (OIM). (2006). Glosario sobre Migración, (Derecho Internacional Sobre Migración núm. 7). https://publications.iom.int/system/files/pdf/iml_7_sp.pdf

Padilla, H. (2012). ¿Repatriado? Una historia de vida y su contexto. En M. D. París (Coord.), Migrantes, desplazados, braceros y deportados. Experiencias migratorias y prácticas políticas (pp. 211-247). El Colegio de la Frontera Norte.

Peña, J. (2017). Realidad frente a hechos alternativos: la migración irregular hacia Estados Unidos y las deportaciones de la población mexicana durante la administración de Trump. Norteamérica, 12(2), 197-212. https://www.sciencedirect. com/science/article/pii/S187035501830034X

Pujadas, J. (1992). Etnografía. Paidós.

Rendón, E. \& Wertman, L. (2017). Reintegración migrante: Un modelo social, económico y empático para el retorno. Cientika. https://www.senado.gob.mx/comisiones/derechos_humanos/docs/Reintegracion_Migrante.pdf

Rivera, L. (2011). ¿Quiénes son los retornados? Apuntes sobre el migrante retornado en el México contemporáneo. En B. Feldman, L. Rivera, C. Stefoni \& M. I. Villa (Comps.). La construcción social del sujeto migrante en América Latina. Prácticas, representaciones y categorías (pp. 309-337). FLACso, clacso, Universidad Alberto Hurtado.

Rivera, L. (2013). Migración de retorno y experiencias de reinserción en la zona metropolitana de la Ciudad de México. Revista Interdisciplinar da Mobilidade Humana, 21(41), 55-76. http://www.scielo.br/pdf/remhu/v21n41/04.pdf

Salas, R, Jardón, A. \& Murguía, V. (2019). La migración internacional de retorno en el Estado de México. Región y Sociedad, 31, 1-24. https://doi.org/10.22198/ rys2019/31/1085

Santamarina, C. \& Marinas, J. M. (1994). Historias de vida e historia oral. En J. M. Delgado \& J. Gutiérrez (Coords.). Métodos y técnicas cualitativas de investigación en Ciencias Sociales (pp. 259-287). Síntesis. 
Sanz, M. (2016). Volver a casa después de vivir en otro país no es tan fácil como parece. Código Nuevo. https://www.codigonuevo.com/mileniales/volver-casa-vivir-facil-parece?fbclid=IwAR0dSik8oD2a5WKuY6CsCuE8CXyxuKYRMDgA3hvarezwTAaybBKoqDFUtqg

Secretaría de Trabajo y Fomento al Empleo del Gobierno de la Ciudad de México, Oficina de la Organización Internacional del Trabajo para México y Cuba. (2018). Programas sociales para población migrante en la Ciudad de México. Identificación de buenas prácticas y recomendaciones en materia de inserción laboral. Secretaría de Trabajo y Fomento al Empleo del Gobierno de la Ciudad de México, Oficina de la Organización Internacional del Trabajo para México y Cuba. https://www.trabajo.cdmx.gob.mx/storage/app/uploads/public/5bc/619/62 1/5bc6196218328636139953.pdf

Taylor, S. \& Bogdan, R. (1984). Introducción a los métodos cualitativos de investigación (pp. 15-99). Paidós.

U.s. Department of State. (2017). Reverse culture shock. https://2009-2017.state.gov$/ \mathrm{m} / \mathrm{fsi} / \mathrm{tc} / \mathrm{c5} 6075 . \mathrm{htm}$

Velasco, L. \& Gianturco, G. (2015). Migración internacional y biografías multiespaciales: una reflexión metodológica. En M. Ariza \& L. Velasco (Coords.), Métodos cualitativos y su aplicación empírica. Por los caminos de la investigación sobre migración internacional (pp. 115-150). Universidad Nacional Autónoma de México, El Colegio de la Frontera Norte.

Zepeda, R. (2016). Determinantes del declive sindical en Estados Unidos. Norteamérica, 11(2), 139-164. https://doi.org/10.20999/nam.2016.b006

Ana Elizabeth Jardón Hernández

Mexicana. Doctora en ciencias sociales por El Colegio de Michoacán. Profesorainvestigadora en el Centro de Investigación en Ciencias Sociales y Humanidades de la Universidad Autónoma del Estado de México. Integrante del Sistema Nacional de Investigadores Nivel 1. Líneas de investigación: migración internacional, retorno y desarrollo. Publicación reciente: Jardón, A. \& Hernández, I. (2019). Dinámica contemporánea de los vínculos transnacionales. Estudio de caso de una comunidad migrante en el sur del Estado de México. Revista Si Somos Americanos, 19(1), 67-84.

Zoraida Ronzón Hernández

Mexicana. Doctora en antropología social por el Centro de Investigaciones y Estudios Superiores en Antropología Social. Profesora-investigadora en el Centro de Investigación en Ciencias Sociales y Humanidades de la Universidad Autónoma del Estado de México. Integrante del Sistema Nacional de Investigadores Nivel 1. Líneas de investigación: vejez y envejecimiento, movilidad humana, género y grupos etarios. Publicación reciente: Ronzón Hernández, Z. \& Méndez Palacios, A. (2019). Procesos de construcción identitaria y resistencia solidaria en personas envejecidas de contextos rurales en México INTEGRA2. Revista Electrónica de Educación Especial y Familia, 10(1), 125-134. 